\title{
Pathways of Adenine Nucleotide Catabolism in Erythrocytes
}

\author{
F. Bontemps, G. Van den Berghe, and H. G. Hers \\ Laboratoire de Chimie Physiologique, Université Catholique de Louvain, and International Institute of Cellular and Molecular Pathology, \\ UCL 75.39, B-1200 Brussels, Belgium
}

\begin{abstract}
The exact pathway whereby the initial catabolism of the adenine nucleotides proceeds from AMP and the possibility of a recycling of adenosine were investigated in human erythrocytes. Adenine nucleotide catabolism, reflected by the production of hypoxanthine, is very slow under physiologic conditions and can be greatly increased by suppression of glucose or alkalinization of the medium. Experiments with inhibitors of adenosine deaminase and adenosine kinase demonstrated that under physiologic conditions the initial catabolism of AMP proceeds by way of a deamination of AMP, followed by dephosphorylation of inosine monophosphate, and that no recycling occurs between AMP and adenosine. Under glucose deprivation, $\sim 75 \%$ of the 20 -fold increase of the catabolism of the adenine nucleotides proceeded by way of a dephosphorylation of AMP followed by deamination of adenosine, and a small recycling of this nucleoside could be evidenced. Inhibition of adenosine transport showed that the dephosphorylation of AMP occurred intracellularly. When the incubation medium was alkalinized in the presence of glucose, the 15-fold increase in the conversion of AMP to hypoxanthine proceeded exclusively by way of AMP deaminase but a small recycling of adenosine could also be evidenced. The threefold elevation of intraerythrocytic inorganic phosphate $\left(\mathbf{P}_{\mathbf{l}}\right)$ during glucose deprivation and its $\mathbf{5 0 \%}$ decrease during alkalinization as well as experiments in which extracellular $\mathbf{P}_{\mathbf{1}}$ was modified, indicate that the dephosphorylation of red blood cell AMP is mainly responsive to variations of AMP, whereas its deamination is more sensitive to $P_{i}$.
\end{abstract}

\section{Introduction}

The catabolism of the adenine nucleotides proceeds from AMP, which is maintained in equilibrium with ATP and ADP by adenylate kinase. As shown in the schematic diagram in Fig. 1, the initial two reactions of this catabolism, i.e., deamination and dephosphorylation, can theoretically occur via two enzyme sequences that both lead to the formation of inosine: on the righthand side of the scheme, deamination by AMP deaminase is followed by dephosphorylation by 5 -nucleotidase, whereas in the left-hand pathway prior dephosphorylation by the $5^{\prime}$-nucleotidase is followed by deamination by adenosine deaminase. The route involving initial dephosphorylation can be interrupted at the level of adenosine which can be reconverted to AMP by

Address reprint requests to Dr. Bontemps, Chimie Physiologique, UCL 75.39, 75 avenue Hippocrate, B-1200 Brussels, Belgium.

Received for publication 19 March 1985 and in revised form 30 November 1985.

J. Clin. Invest.

(C) The American Society for Clinical Investigation, Inc.

0021-9738/86/03/0824/07 \$1.00

Volume 77, March 1986, 824-830 adenosine kinase (step 5 in Fig. 1). This results in a substrate cycle between AMP and adenosine, the operation of which has been well documented in the liver (1). The inosine formed is finally split by phosphorolysis into ribose 1-phosphate and hypoxanthine, which in most human tissues, including erythrocytes, constitutes the terminal product of the pathway, as significant amounts of xanthine oxidase, which catalyzes the formation of uric acid, are only found in liver and small intestinal mucosa (2).

The conservation of the adenine nucleotide pool requires a strict control of both degradative pathways. This can be achieved by a limitation of the activity of the two AMP-degrading enzymes and by recovery of adenosine through adenosine kinase. The mechanisms that keep the catabolism of erythrocytic AMP to a minimum under physiologic conditions have not been completely unraveled. Inhibition of red cell AMP deaminase by inorganic phosphate $\left(\mathrm{P}_{\mathrm{i}}\right)^{1}$ and 2,3-bisphosphoglycerate (2,3-DPG) has been reported (3-6), but the control of the dephosphorylation of inosine monophosphate (IMP) and AMP remains unexplored because of the lack of knowledge concerning the enzyme(s) catalyzing this process. Indeed, although lysates of human erythrocytes have been shown to dephosphorylate pyrimidine 5'-nucleotides, no activity has been observed with purine 5 '-nucleotides $(7,8)$.

Little is known also concerning the respective participation of the two enzyme sequences shown in Fig. 1 in the catabolism of adenine nucleotides and the occurrence of recycling of adenosine in erythrocytes. These questions can be addressed with inhibitors of adenosine metabolism. Inhibition of adenosine deaminase will selectively decrease the production of hypoxanthine resulting from the dephosphorylation of AMP. On the other hand, inhibition of adenosine kinase will increase the production of hypoxanthine if recycling of adenosine has a significant role. The inhibition of both enzymes should produce accumulation of adenosine. A few studies have assessed the involvement of deamination and dephosphorylation of AMP in the breakdown of erythrocytic ATP induced by various drugs and metabolic inhibitors $(9,10)$. The catabolism of the adenine nucleotides under physiologic conditions and its acceleration provoked by lack of glucose (11), alkalinization of the medium $(12,13)$, and depletion of $P_{i}$ (14-16) have, however, not been investigated in this respect, nor has the possibility of a recycling of adenosine been evaluated.

In the present study of human erythrocytes, as in previous work in isolated rat hepatocytes $(1,17,18)$, the pathway of the degradation of the adenine nucleotides was investigated after prior labeling of the adenine nucleotide pool with $\left[{ }^{14} \mathrm{C}\right]$ adenine. The rate of appearance of radioactivity in the purine catabolites

1. Abbreviations used in this paper: $\mathrm{dCF}$, deoxycoformycin; 2.3-DPG, 2,3-bisphosphoglycerate; F-1,6-DP, fructose 1,6-bisphosphate; IMP, inosine monophosphate; ITu, iodotubercidin; KRB, Krebs-Ringer bicarbonate (buffer); $\mathbf{P}_{i}$, inorganic phosphate. 


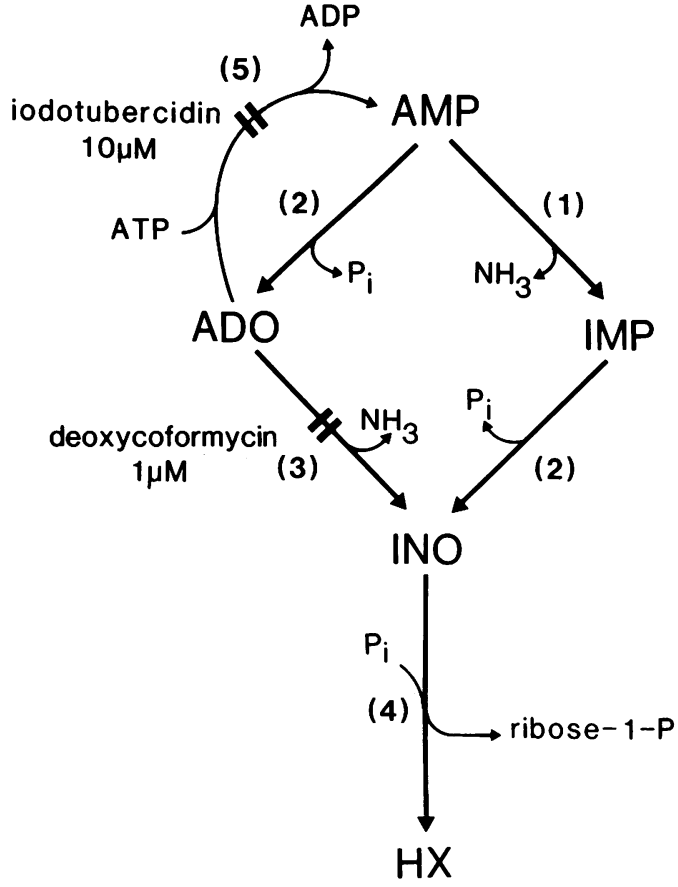

Figure 1. The pathway of the degradation of adenine nucleotides in human erythrocytes: (1) AMP deaminase, (2) 5'-nucleotidase, (3) adenosine deaminase, (4) nucleoside phosphorylase, (5) adenosine kinase. ADO, adenosine; HX, hypoxanthine; INO, inosine.

was followed in the absence and in the presence of inhibitors of adenosine metabolism. Deoxycoformycin (dCF) rather than coformycin was used to inhibit adenosine deaminase because of its higher potency at low concentrations (19). As previously reported (1) iodotubercidin (ITu) was used to inhibit adenosine kinase (20).

\section{Methods}

Materials. $\left[\mathrm{U}-{ }^{14} \mathrm{C}\right]$ adenine $(296 \mathrm{Ci} / \mathrm{mol})$ and $\left[8-{ }^{14} \mathrm{C}\right]$ adenosine $(56$ $\mathrm{Ci} / \mathrm{mol}$ ) were purchased from The Radiochemical Centre (Amersham, Buckinghamshire, England). dCF was from Warner Lambert (Detroit, MI), and ITu was a gift of Dr. L. B. Townsend (University of Michigan College of Pharmacy, Ann Arbor, MI). The adenosine transport inhibitor R-51469 (Mioflazine) was a gift of Dr. H. Van Belle (Janssen Pharmaceutica, Beerse, Belgium). Silicone (Siliconöl AR 200, 200 centistoke) was obtained from Serva (Heidelberg, Federal Republic of Germany). The source of all other chemicals has been given previously (17).

Incubations. Fresh blood, taken from a cubital vein of healthy human volunteers, was collected on heparin. Plasma and buffy coat were removed by centrifugation at room temperature. The red cells were washed three times with Krebs-Ringer bicarbonate (KRB) buffer, $\mathrm{pH} 7.4$, containing $5 \mathrm{mM}$ glucose and gassed with $95 \% \mathrm{O}_{2}-5 \% \mathrm{CO}_{2}(21)$. They were then resuspended, unless otherwise noted, in the same medium as a $20 \%$ hematocrit. Labeling of the erythrocytic adenine nucleotide pool was performed by preincubating the cells with $1 \mu \mathrm{M}\left[\mathrm{U}-{ }^{14} \mathrm{C}\right]$ adenine in a gassed, stoppered flask for $60 \mathrm{~min}$ at $37^{\circ} \mathrm{C}$ in a shaking water bath. This was followed by two washes and resuspension of the cells as a $20 \%$ hematocrit in KRB buffer or modifications thereof, with or without inhibitors, as described under results. $3-5 \mathrm{ml}$ of the cell suspension was incubated at $37^{\circ} \mathrm{C}$ with shaking in tightly stoppered siliconized vials. To avoid modifications of the $\mathrm{pH}$ of the incubation medium by $\mathrm{CO}_{2}$ loss, the vials were carefully regassed after removal of each aliquot.

Extraction procedures. For analytical determinations on the red cell suspension as a whole, $0.5-\mathrm{ml}$ aliquots were transferred into $0.25 \mathrm{ml}$ of ice-cold $10 \% \mathrm{HClO}_{4}$ at the times indicated. After centrifugation, the resulting supernatants were neutralized with $3 \mathrm{M} \mathrm{KOH} / 3 \mathrm{M} \mathrm{KHCO}_{3}$, and reclarified by centrifugation. For separate analysis of the medium and of the red cell content, $0.5 \mathrm{ml}$ of cell suspension was centrifuged through a $0.5-\mathrm{ml}$ layer of silicone into $0.5 \mathrm{ml}$ of ice-cold $10 \% \mathrm{HClO}_{4}$ as described by Klingenberg and Pfaff (22) with the modifications of Whelan and Bagnara (23).

Assessment of inhibitor requirements. The concentration of dCF required to suppress the activity of adenosine deaminase in intact erythrocytes was determined by the ability to inhibit the conversion of $\left[{ }^{14} \mathrm{C}\right]$ adenosine, added to the cell suspension, into inosine and hypoxanthine. At $1 \mu \mathrm{M}, \mathrm{dCF}$ inhibited the deamination of $100 \mu \mathrm{M}$ adenosine by $95 \%$ and that of $10 \mu \mathrm{M}$ adenosine by $98 \%$. The concentration of ITu needed to abolish adenosine kinase activity was determined by its capacity to inhibit the incorporation of $\left[{ }^{14} \mathrm{C}\right]$ adenosine into the erythrocytic adenine nucleotides. $10 \mu \mathrm{M}$ ITu inhibited the incorporation of $10 \mu \mathrm{M}$ adenosine by $98 \%$. The effect of R-51469 on the transport of adenosine was measured at $20^{\circ} \mathrm{C}$ in erythrocytes that had been preincubated for $15 \mathrm{~min}$ in the presence of $10 \mu \mathrm{M}$ ITu and $1 \mu \mathrm{M} \mathrm{dCF}$ in order to inhibit the metabolism of the nucleoside. Influx was calculated by determining, $5 \mathrm{~min}$ after the addition of $100 \mu \mathrm{M}\left[{ }^{14} \mathrm{C}\right]$ adenosine, the radioactivity in the pellet obtained after centrifugation of the cell suspension through silicone. To measure the efflux of adenosine, erythrocytes were equilibrated with $100 \mu \mathrm{M}\left[{ }^{14} \mathrm{C}\right]$ adenosine for $30 \mathrm{~min}$, pelleted by centrifugation, and thereafter diluted fivefold in adenosine-free medium. After $5 \mathrm{~min}$, the cells were centrifuged through silicone and the radioactivity of the extracellular medium was determined. At a concentration of $10 \mu \mathrm{M}$, $\mathrm{R}-51469$ inhibited the influx as well as the efflux of $100 \mu \mathrm{M}$ adenosine from erythrocytes by $99 \%$.

Analytical methods. The concentrations of ATP, ADP, AMP, and IMP were measured by high-pressure anion-exchange chromatography (24). The radioactivity in the adenine nucleotides was determined after their one-dimensional separation on polyethyleneimine-cellulose thinlayer plates, and that in adenosine, inosine, adenine, and hypoxanthine after chromatography on cellulose thin-layer plates (25). The concentrations of the latter compounds were calculated from the specific radioactivity of the adenine nucleotides, which remained constant over the duration of the experiments. $\mathrm{P}_{\mathrm{i}}$ was measured according to Itaya and $\mathrm{Ui}$ (26), lactate according to Gawehn and Bergmeyer (27), 2,3-DPG according to Michal (28), and fructose 1,6-bisphosphate (F-1,6-DP) and the triose phosphates according to Michal and Beutler (29).

Results are expressed as means \pm standard error of the mean. Differences between experimental conditions were tested for significance using the paired $t$ test.

\section{Results}

Degradation of erythrocytic adenine nucleotides under physiologic conditions. Erythrocytes incubated in KRB containing $5 \mathrm{mM}$ glucose and $1.2 \mathrm{mM} P_{i}$ maintained constant adenine nucleotide levels for several hours. They produced only a small amount of hypoxanthine $(4.2 \pm 0.7 \mathrm{nmol} / \mathrm{h}$ per $\mathrm{ml}$ of packed cells; mean \pm SEM of eight experiments), corresponding to a loss of $0.3 \%$ of the adenine nucleotide pool per hour. No adenosine, inosine, or adenine could be detected in the cell suspension. Inhibition of adenosine deaminase by $\mathrm{dCF}$ did not significantly modify these results, indicating that under physiologic conditions, the formation of hypoxanthine results from the deamination of AMP followed by the dephosphorylation of IMP. No recycling of adenosine could be evidenced in that inhibition of adenosine kinase by ITu did not increase the production of hypoxanthine during the same time interval. Likewise, the addition of both inhibitors together was without significant effect.

Degradation of the adenine nucleotides induced by glucose deprivation. Incubation of the erythrocytes in KRB buffer devoid 
of glucose resulted in drastic modifications of their adenine nucleotide concentrations (expressed as micromoles per milliliter of packed cells). ATP fell from 1.2 to 0.25 after $4 \mathrm{~h}$ (Fig. $2 a$ ), while ADP increased from 0.15 to 0.40 (Fig. $2 b$ ) and AMP from 0.01 to 0.30 (Fig. $2 c$ ) over the same time interval. IMP increased only from 0.01 to 0.04 (Fig. $2 d$ ). There was an approximate 20-fold increase of the production of hypoxanthine as compared to control conditions (Fig. $2 e$ ), to $100 \mathrm{nmol} / \mathrm{h}$ per ml of packed cells in the experiment depicted. Accumulated hypoxanthine accounted quantitatively for the loss of adenine nucleotides. Indeed, after $4 \mathrm{~h}$, when about one-third of the adenine nucleotide pool, or $0.4 \mu \mathrm{mol} / \mathrm{ml}$ of packed cells was lost (Fig. $2 d$ ), the concentration of the purine base reached $\sim 0.08 \mu \mathrm{mol} / \mathrm{ml}$ in the 1:5 cell suspension. Experiments in which the concentration of glucose was varied from 0.5 to $5 \mathrm{mM}$ demonstrated that the rate of production of hypoxanthine increased only when glucose had been completely utilized (not illustrated).

The increased rate of catabolism of the adenine nucleotides provoked by lack of glucose obviously resulted from the 30 -fold elevation of the concentration of AMP, which is the substrate of both AMP deaminase and 5 '-nucleotidase. The inhibition of adenosine deaminase by $1 \mu \mathrm{M}$ dCF had several effects on this catabolism. The most important one was a 75\% decrease in the rate of production of hypoxanthine, from 100 to $\sim 25 \mathrm{nmol} / \mathrm{h}$ per $\mathrm{ml}$ of packed cells (Fig. $2 e$ ). This indicates that during glucose deprivation, one-fourth of AMP degradation goes through the sequence AMP deaminase/5'-nucleotidase, and three-fourths via the sequence 5 '-nucleotidase/adenosine deaminase. Accordingly, the decrease in the production of hypoxanthine was accompanied by an accumulation of adenosine (Fig. $2 f$ ). However, this accumulation of adenosine did not account for the decrease in production of hypoxanthine because the production of total purine catabolites was diminished, particularly during the first hour of incubation (Fig. $2 h$ ). Yet, if both adenosine deaminase and adenosine kinase were inhibited by dCF and ITu together, adenosine accumulated from the beginning of the experiment (Fig.

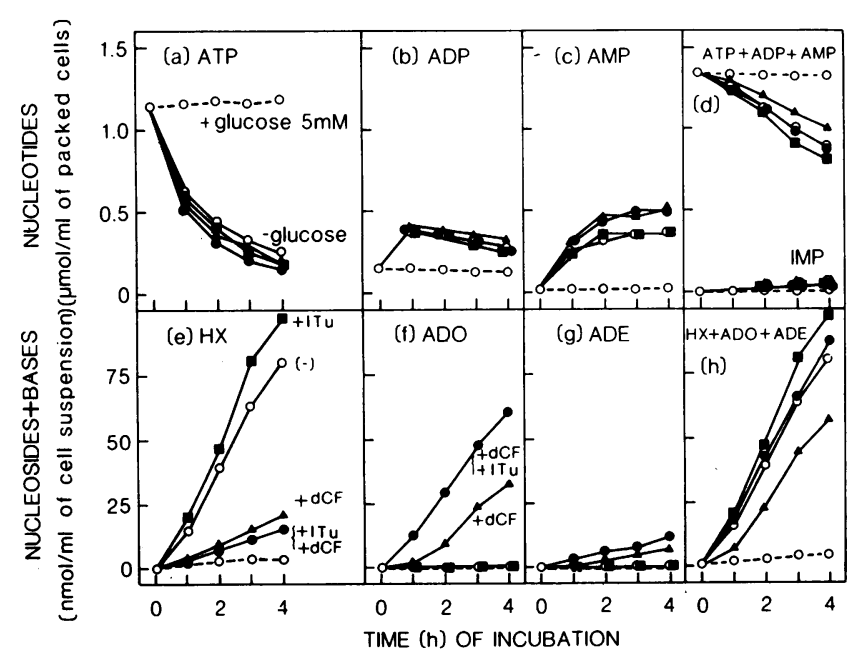

Figure 2. Effect of lack of glucose on the catabolism of the erythrocytic adenine nucleotides. After two washes performed at $20^{\circ} \mathrm{C}$ in glucose-free medium, the cells were resuspended in KRB in the presence (dashed lines) or in the absence (solid lines) of glucose, without inhibi-

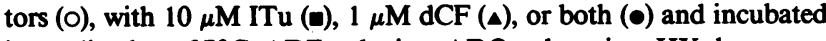
immediately at $37^{\circ} \mathrm{C}$. ADE, adenine; $\mathrm{ADO}$, adenosine; $\mathrm{HX}$, hypoxanthine.
$2 f$ ) and the production of total purine catabolites was the same as in the absence of inhibitors (Fig. $2 h$ ). These findings indicate that in the presence of $\mathrm{dCF}$ alone, adenosine was partly rephosphorylated by adenosine kinase, particularly during the first hour of incubation, when the concentration of ATP was still above $0.5 \mathrm{mM}$ (Fig. $2 a$ ). The reduction in the rate of production of total purine catabolites during this time interval, reaching $\sim 50 \mathrm{nmol} / \mathrm{h}$ per $\mathrm{ml}$ of packed cells, reflects the magnitude of the rate of rephosphorylation of adenosine. Recycling of adenosine in the presence of $\mathrm{dCF}$ alone is further corroborated by the noticeable protection of the adenine nucleotide pool under this condition (Fig. $2 d$ ).

That some recycling of adenosine also occurs in the absence of $\mathrm{dCF}$ is indicated by the slight but reproducible effect of ITu alone, to increase hypoxanthine formation by $15-20 \%$ (from 100 to $120 \mathrm{nmol} / \mathrm{h}$ per $\mathrm{ml}$ of packed cells in Fig. $2 e$ ). The lower rate of recycling in the absence of $\mathrm{dCF}(20 \mathrm{nmol} / \mathrm{h}$ per $\mathrm{ml}$ of packed cells, as compared to 50 in the presence of the inhibitor) can be explained by a competition of adenosine deaminase for the metabolism of adenosine by adenosine kinase in the former condition.

Another fact apparent in glucose deprivation is the formation of a small amount of adenine (Fig. $2 \mathrm{~g}$ ). It is, however, limited to conditions in which adenosine accumulates. This in agreement with previous observations made by Mills et al. (10). As discussed by these authors, it is most likely explained by a phosphorolysis of adenosine favored by the elevation of $P_{i}$. As shown in Table $\mathrm{I}$, deprivation of glucose for $2 \mathrm{~h}$ resulted in an approximate threefold increase, from 0.9 to $2.4 \mu \mathrm{mol} / \mathrm{ml}$ of packed cells, of the concentration of $P_{i}$ inside the erythrocytes and in a $25 \%$ decrease, from 4.2 to $3.1 \mu \mathrm{mol} / \mathrm{ml}$ of packed cells, of that of 2,3-DPG.

In order to localize the site of formation of adenosine in the erythrocytes, experiments were performed with the nucleoside transport inhibitor R-51469. The influence of this drug on the intra- and extracellular accumulation of adenosine induced by glucose deprivation in the presence of ITu and dCF is shown in Fig. 3. Whereas the concentration of adenosine was approximately the same inside and outside the erythrocytes in the control experiment, addition of R-51469 induced a marked accumulation intracellularly, with decreased external concentrations over $3 \mathrm{~h}$. After $1 \mathrm{~h}$ of incubation, the concentration of adenosine inside the cells was $21 \mathrm{nmol} / \mathrm{ml}$ as compared to $3 \mathrm{nmol} / \mathrm{ml}$ in the medium. The other modifications of the adenine compounds induced by glucose deprivation, as well as the intra- and extracellular accumulation of hypoxanthine, were not influenced by

Table I. Effect of Lack of Glucose on the Concentrations of $P_{i}$ and of 2,3-DPG inside Erythrocytes

\begin{tabular}{lll}
\hline Conditions & $\mathrm{P}_{\mathbf{i}}$ & $2,3-\mathrm{DPG}$ \\
\hline & $\mu \mathrm{mol} / \mathrm{ml}$ of packed cells & $\mu \mathrm{mol} / \mathrm{ml}$ of packed cells \\
& & \\
With glucose & $0.88 \pm 0.05$ & $4.17 \pm 0.44$ \\
No glucose & $2.42 \pm 0.15^{*}$ & $3.07 \pm 0.44^{*}$
\end{tabular}

Erythrocytes were resuspended in the presence or in the absence of 5 $\mathrm{mM}$ glucose and incubated for $2 \mathrm{~h}$ before the determinations. Values are means \pm SEM from four experiments ${ }^{*} P<0.01$. 


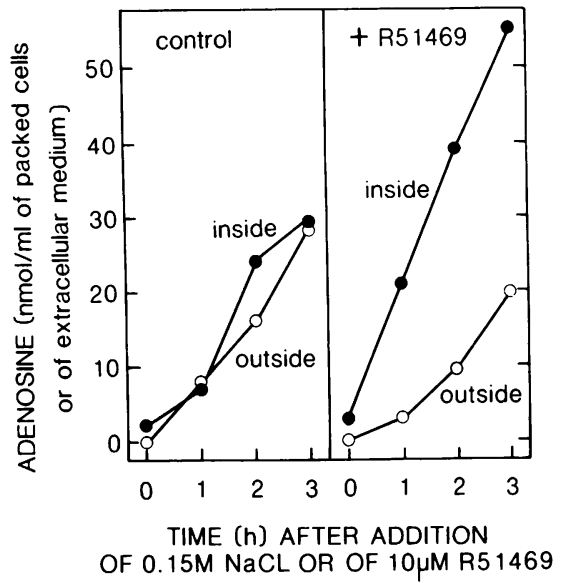

Figure 3. Influence of R-51469 on the intracellular concentration of adenosine. After two washes in KRB without glucose, the erythrocytes were resuspended in the same medium containing $1 \mu \mathrm{M} \mathrm{dCF}$ and 10 $\mu \mathrm{M}$ ITu. After $15 \mathrm{~min}$ of incubation, $\mathrm{NaCl}$ or $10 \mu \mathrm{M}$ R-51469 were added to the cell suspension.

R-51469 (results not shown). All these results indicate that adenosine was formed intracellularly by a cytosolic enzyme.

Degradation of the adenine nucleotides provoked by alkalinization of the medium. Incubation of the erythrocytes in KRB containing $5 \mathrm{mM}$ glucose and $50 \mathrm{mM} \mathrm{NaHCO}$ (instead of the physiologic concentration of $25 \mathrm{mM}$ ), resulting in a pH of about 7.7, provoked a loss of ATP, which was less marked than with glucose deprivation since its concentration decreased only from 1 to $0.7 \mu \mathrm{mol} / \mathrm{ml}$ of packed cells, and ceased after $2 \mathrm{~h}$ (Fig. $4 a$ ). There was a concomitant increase in the concentration of AMP, which reached $0.05 \mu \mathrm{mol} / \mathrm{ml}$ of packed cells, or approx-

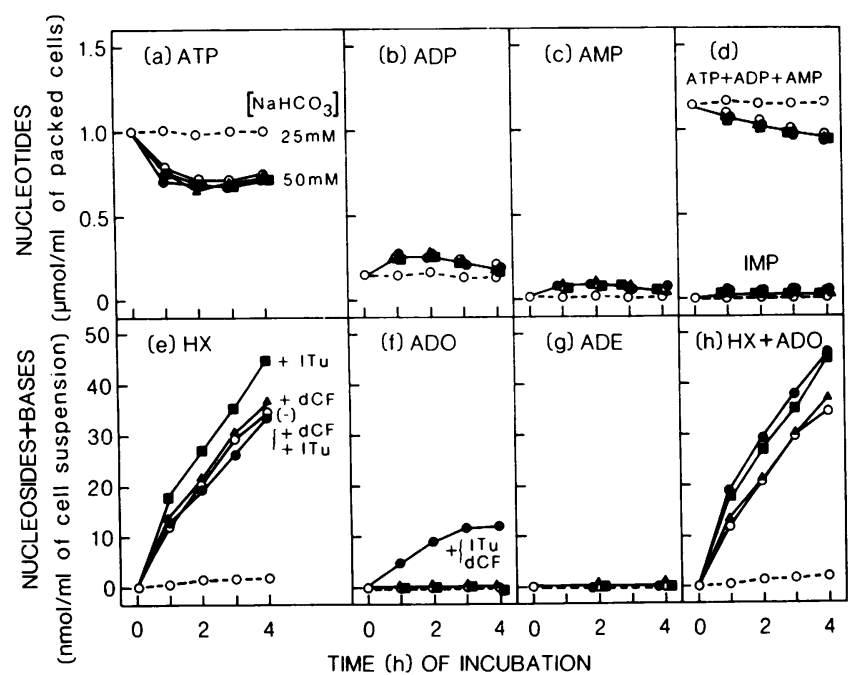

Figure 4. Effect of alkalinization of the incubation medium on the catabolism of the adenine nucleotides. The cells were resuspended in the presence of glucose in the usual KRB buffer in which the concentration of $\mathrm{NaHCO}_{3}$ is $25 \mathrm{mM}$ (dashed lines) or in a modified $\mathrm{KRB}$ in which the concentration of $\mathrm{NaHCO}_{3}$ was increased to $50 \mathrm{mM}$ and the concentration of $\mathrm{NaCl}$ commensurately decreased (solid lines). They were incubated without inhibitors (0) or with $10 \mu \mathrm{M} \mathrm{ITu} \mathrm{(}), 1 \mu \mathrm{M}$ dCF (४), or both (๑). ADE, adenine; ADO, adenosine; HX, hypoxanthine. imately fivefold its initial value after $1 \mathrm{~h}$, and decreased progressively after $2 \mathrm{~h}$ (Fig. $4 \mathrm{c}$ ). The concentration of IMP did not increase above $10 \mu \mathrm{M}$ (Fig. $4 d$ ). This decrease in ATP and increase in AMP are currently believed $(12,30-32)$ to be caused by an imbalance between the first half of glycolysis which consumes ATP and is accelerated by alkalinization, and the second half which produces ATP. As shown in Table II, this imbalance is evidenced by an accumulation of the glycolytic intermediates, F-1,6-DP and the triose phosphates. The concentrations of these metabolites were increased more than 50 -fold, to 0.5 and 0.8 $\mu \mathrm{mol} / \mathrm{ml}$ of packed cells respectively, in agreement with previous findings (12, 30-32). In marked contrast with the effect of glucose deprivation in increasing the intracellular concentration of $P_{i}$, alkalinization lowered its concentration by more than $50 \%$, to $\sim 0.4 \mu \mathrm{mol} / \mathrm{ml}$ of packed cells. Alkalinization did not modify significantly the concentration of 2,3-DPG (Table II).

As in glucose deprivation, the rise in AMP concentration was accompanied by a loss of the total adenine nucleotide pool reaching $0.2 \mu \mathrm{mol} / \mathrm{ml}$ of packed cells after $4 \mathrm{~h}$ (Fig. $4 d$ ). This loss was quantitatively recovered under the form of nearly 0.04 $\mu \mathrm{mol}$ of hypoxanthine per $\mathrm{ml}$ of cell suspension (Fig. $4 e$ ). The rate of formation of hypoxanthine was elevated 10-15-fold as compared to cells incubated in $25 \mathrm{mM} \mathrm{NaHCO}$, to $50 \mathrm{nmol} /$ $\mathrm{h}$ per $\mathrm{ml}$ of packed cells in the experiment shown.

As in the preceding section, the two inhibitors, dCF and ITu, were used in order to clarify the pathway by which the conversion of adenine nucleotides to hypoxanthine proceeded during alkalinization. In sharp contrast with what had been observed in glucose deprivation, $\mathrm{dCF}$ alone had no effect on this conversion (Fig. $4 e$ ). This rules out the participation of adenosine deaminase in the formation of hypoxanthine, which therefore proceeds exclusively by way of AMP deaminase. However, as in glucose deprivation, ITu alone increased the production of hypoxanthine by $20 \mathrm{nmol} / \mathrm{h}$ per $\mathrm{ml}$ of packed cells (Fig. $4 e$ ). This increase was suppressed if dCF was added together with ITu, and replaced by an equivalent production of adenosine (Fig. $4 f$ ). These observations indicate that in the absence of adenosine kinase inhibition, adenosine was actually formed from AMP by 5'-nucleotidase at the rate of $20 \mathrm{nmol} / \mathrm{h}$ per $\mathrm{ml}$ of packed cells, but completely rephosphorylated by adenosine kinase, with no conversion to hypoxanthine. In accordance with the low $P_{i}$ content of erythrocytes, no adenine was formed when adenosine accumulated (Fig. 4 g).

Table II. Effect of Alkalinization of the Medium on the Concentrations of Metabolites inside Erythrocytes

\begin{tabular}{|c|c|c|c|c|}
\hline Conditions & $\mathbf{P}_{\mathbf{i}}$ & 2,3-DPG & $\begin{array}{l}\text { Triose } \\
\text { phosphate }\end{array}$ & F-1,6-DP \\
\hline & \multicolumn{4}{|c|}{$\mu \mathrm{mol} / \mathrm{ml}$ of packed cells } \\
\hline $\begin{array}{r}\mathrm{NaHCO}_{3} \\
25 \mathrm{mM}\end{array}$ & $0.95 \pm 0.02$ & $3.72 \pm 0.20$ & $0.002 \pm 0.001$ & $0.008 \pm 0.002$ \\
\hline $\begin{array}{r}\mathrm{NaHCO}_{3} \\
50 \mathrm{mM}\end{array}$ & $0.43 \pm 0.02^{*}$ & $3.86 \pm 0.15$ & $0.788 \pm 0.043^{*}$ & $0.508 \pm 0.061^{*}$ \\
\hline
\end{tabular}

Erythrocytes were resuspended in the presence of glucose in the usual or in the alkalinized KRB buffer and incubated for $2 \mathrm{~h}$ before the determinations. Values are means \pm SEM from four experiments.

${ }^{*} P<0.01$ 
Influence of the concentration of $P_{i}$ in the incubation medium. $P_{i}$ is a known inhibitor of both AMP deaminase $(3,5,6,33)$ and cytoplasmic 5 -nucleotidase $(34,35)$ of various origins. The observations that the concentration of $\mathbf{P}_{\mathbf{i}}$ in the erythrocytes was increased in glucose deprivation and decreased after alkalinization, prompted a study of the effects of variations of $P_{i}$ in the extracellular medium on the production of hypoxanthine. To distinguish between the effects of $P_{i}$ on 5 -nucleotidase and on AMP deaminase, experiments were also performed in the presence of dCF and ITu together. In this condition, the production of adenosine reflects the activity of 5 -nucleotidase and the residual formation of hypoxanthine that of AMP deaminase.

As shown in Fig. $5 a$, at physiologic pH and in the presence of $5 \mathrm{mM}$ glucose, suppression of $P_{i}$ in the incubation medium resulted in an approximate fourfold enhancement of the rate of production of hypoxanthine, from 4 to $15 \mathrm{nmol} / \mathrm{h}$ per $\mathrm{ml}$ of packed cells. Elevation of the concentration of $P_{i}$ from its control value of $1.2 \mathrm{mM}$ to $10 \mathrm{mM}$, abolished the production of the purine base. Because of the very low rates of catabolism in the presence of glucose, no significant changes in the concentrations of ATP were noticed over the duration of the experiment. An approximate twofold increase in AMP, from 10 to $20 \mu \mathrm{mol} / \mathrm{ml}$ of packed cells in the absence of $\mathrm{P}_{i}$, and a $50 \%$ decrease in the presence of $10 \mathrm{mM} P_{i}$, were nevertheless recorded (not illustrated) and most likely contribute to the observed modifications of the rate of production of hypoxanthine. In the presence of $\mathrm{dCF}$ and ITu together, the rates of production of hypoxanthine

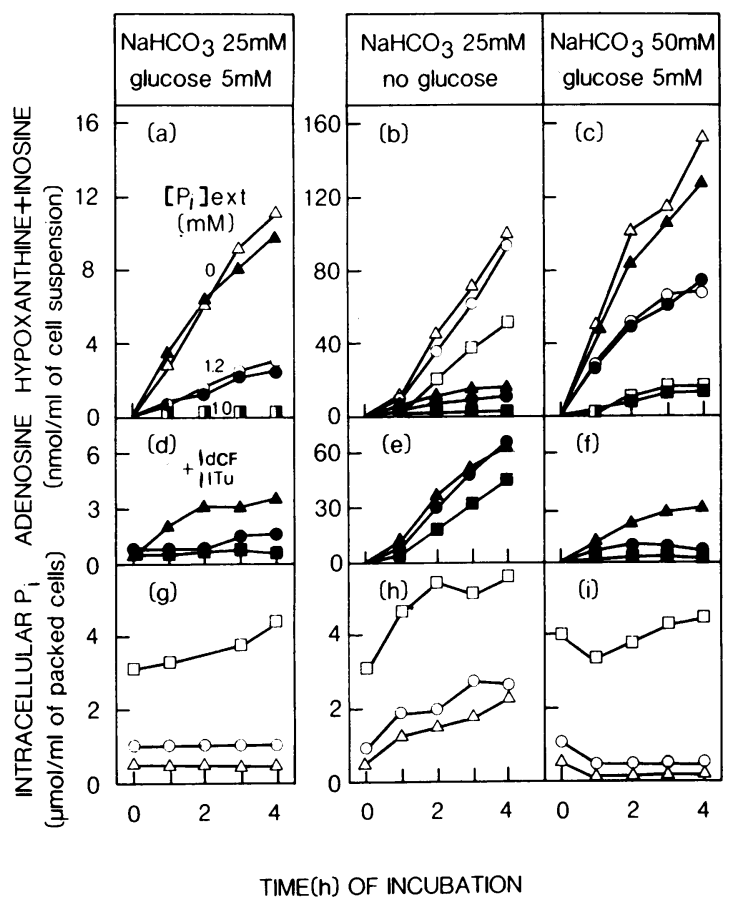

Figure 5. Effect of the concentration of $\mathrm{P}_{\mathrm{i}}$ on the catabolism of the adenine nucleotides. Preincubation of the erythrocytes with $\left[{ }^{14} \mathrm{C}\right]$ adenine, and incubations were performed in the presence of three concentrations of $P_{i}: 0 \mathrm{mM}(\Delta, \Delta), 1.2 \mathrm{mM}(0, \bullet)$, or $10 \mathrm{mM}(\square, \square)$. The concentration of $\mathrm{Ca}^{++}$in the $\mathrm{KRB}$ buffer was also reduced from 2.5 to $1.25 \mathrm{mM}$. The incubation medium contained $5 \mathrm{mM}$ glucose (left and right panels) or not (middle panels) or was alkalinized (right panels). Erythrocytes were incubated without (open symbols) or with (closed symbols) $10 \mu \mathrm{M}$ ITu and $1 \mu \mathrm{M}$ dCF. Inosine was detected only at alkaline $\mathrm{pH}$ and in the absence of extracellular $\mathrm{P}_{\mathrm{i}}$ and amounted then to $60 \%$ of the sum of hypoxanthine and inosine. were not modified, but in the absence of extracellular $\mathbf{P}_{i}$ a small accumulation of adenosine, to $3 \mathrm{nmol} / \mathrm{ml}$ of packed cell suspension, could be evidenced (Fig. $5 d$ ). This indicates that cytoplasmic 5'-nucleotidase acting on AMP became slightly active under this condition. Determination of the intracellular concentration of $\mathrm{P}_{\mathrm{i}}$ (Fig. $5 \mathrm{~g}$ ) showed that it had been decreased from 1 to $0.6 \mu \mathrm{mol} / \mathrm{ml}$ of cell suspension by preincubation in the absence of $P_{i}$, had been elevated threefold by incubation in the presence of $10 \mathrm{mM} P_{i}$, and continued to increase over the duration of the experiment in the latter condition.

As shown in Fig. $5 b$, the high rate $(\sim 100 \mathrm{nmol} / \mathrm{h}$ per $\mathrm{ml}$ of packed cells) of hypoxanthine production induced by the suppression of glucose (a condition in which intracellular $P_{i}$ is elevated approximately threefold) was markedly less influenced by modifications of the concentration of $P_{i}$ in the incubation medium. Withdrawal of $P_{i}$ had very little effect on the rate of production of hypoxanthine, and incubation in $10 \mathrm{mM} \mathrm{P} \mathrm{P}_{\mathrm{i}}$ only resulted in a $40 \%$ reduction of the formation of the purine base. The effects on the already large production of adenosine $(\sim 80$ $\mathrm{nmol} / \mathrm{h}$ per $\mathrm{ml}$ of packed cells) recorded upon addition of $\mathrm{dCF}$ and ITu were similarly small (Fig. $5 e$ ). In contrast, the residual rate of formation of hypoxanthine measured under this condition ( $\sim 15 \mathrm{nmol} / \mathrm{h}$ per $\mathrm{ml}$ of packed cells) and believed to occur via AMP deaminase, was approximately doubled in the absence of $P_{i}$ and reduced by $\sim 75 \%$ in the presence of $10 \mathrm{mM} P_{i}$ (Fig. 5 $b$ ). At the three levels of extracellular $P_{i}$, approximately parallel increases in the intracellular concentration of this ion were recorded (Fig. $5 h$ ). The loss of the adenine nucleotides was not modified by the suppression of $\mathrm{P}_{\mathrm{i}}$, but, as expected from the decreased formation of hypoxanthine, was slightly reduced at $10 \mathrm{mM} \mathrm{P}_{\mathrm{i}}$ (results not shown).

At alkaline $\mathrm{pH}$ (a condition that lowers intracellular $\mathrm{P}_{\mathrm{i}}$ ) as in physiologic conditions, modifications of the extracellular concentration of $P_{i}$ markedly influenced the catabolism of the erythrocytic adenine nucleotides (Fig. 5, right panels). In the absence of $P_{i}$, for reasons explained below, the formation of hypoxanthine was accompanied by a nearly equivalent production of inosine so that the rate of production of purine catabolites was doubled, from 100 to $200 \mathrm{nmol} / \mathrm{h}$ per $\mathrm{ml}$ of packed cells (Fig. $5 \mathrm{c}$ ). In the presence of $10 \mathrm{mM} \mathrm{P}_{\mathrm{i}}$, the production of hypoxanthine decreased by $>80 \%$. These effects were observed in the absence as well as in the presence of dCF and ITu. The limited accumulation of adenosine, to $\sim 10 \mathrm{nmol} / \mathrm{ml}$ of cell suspension, recorded under the latter condition was also increased two- to threefold by removal of $P_{i}$, and nearly suppressed by its elevation to $10 \mathrm{mM}$ (Fig. $5 \mathrm{f}$ ). A decrease of similar magnitude of the intracellular concentration of $\mathrm{P}_{\mathrm{i}}(\sim 0.5 \mu \mathrm{mol} / \mathrm{ml}$ of packed cells) was noted during the first hour of incubation at the three levels of extracellular $\mathrm{P}_{\mathrm{i}}$ (Fig. $5 i$ ). Decreased activity of nucleoside phosphorylase, owing to the very low intracellular $P_{i}$ concentrations recorded in the $\mathrm{P}_{\mathrm{i}}$-free medium (around $0.2 \mu \mathrm{mol} / \mathrm{ml}$ of packed cells), is the most likely explanation of the accumulation of inosine observed under this condition. As expected, the loss of adenine nucleotides was increased twofold by the removal of $P_{i}$ and nearly suppressed at $10 \mathrm{mM} P_{i}$ (not illustrated). The more rapid loss of ATP in the absence of $P_{i}$ was accompanied by an approximate twofold increase in AMP, and the slowdown of the ATP depletion at $10 \mathrm{mM} P_{i}$ was associated with a $70 \%$ reduction of the elevation of AMP (not illustrated). The rate of glycolysis which is increased approximately twofold at alkaline $\mathrm{pH}$ as compared to physiologic conditions (30-32), was reduced by $30 \%$ in $P_{i}$-free medium and increased by the same value at 10 $\mathrm{mM} \mathrm{P}_{\mathrm{i}}$ (results not shown). 


\section{Discussion}

Thanks to the use of specific inhibitors of adenosine deaminase and adenosine kinase, we have been able to demonstrate that both enzyme sequences, as diagramed in Fig. 1, can intervene in the initial degradation of erythrocytic AMP, and that their relative participation varies according to the experimental conditions. As already discussed in Results, our data allow us to conclude that the very slow physiologic catabolism of the adenine nucleotides ( $\sim 4 \mathrm{nmol} / \mathrm{h}$ per $\mathrm{ml}$ of packed cells) and its marked, 10-15-fold acceleration induced by alkalinization of the medium, occur by deamination of AMP followed by dephosphorylation of IMP. In this respect, erythrocytes behave like hepatocytes. Indeed, in the latter cells, the physiologic catabolism of the adenine nucleotides and its increase induced by fructose (17) or by anoxia (18) proceed exclusively by this pathway. In contrast, only $25 \%$ of the rapid, 20 -fold accelerated degradation of adenine nucleotides resulting from glucose deprivation in human erythrocytes proceeds by way of AMP deaminase; the remaining $75 \%$ occurs by prior dephosphorylation of AMP into adenosine, followed by deamination of adenosine. Our observation that adenosine accumulates intracellularly in the presence of an inhibitor of nucleoside transport (Fig. 3) demonstrates that human erythrocytes contain a cytosolic 5 '-nucleotidase. We have been able to isolate this enzyme and to separate it from pyrimidine 5'nucleotidase and from acid phosphatase (36).

Our studies also provide an insight in the regulation of both AMP-degrading enzymes inside the human erythrocyte. The activity of the cytoplasmic 5 '-nucleotidase is reflected by the accumulation of adenosine in the presence of dCF and ITu together. As shown in Fig. 5, the 5'-nucleotidase is strongly dependent upon the concentration of AMP: the rates of accumulation of adenosine, recorded under glucose deprivation (Fig. $5 e$ ) a situation in which AMP increases 30 -fold, are several fold higher than in alkalinization (Fig. $5 f$ ), during which AMP increases only fivefold. As apparent from the influence of modifications of extracellular $P_{i}$ on the production of adenosine, the 5 '-nucleotidase is inhibited by $P_{i}$. Yet this inhibitory effect is markedly dependent on the concentration of AMP: at the high concentrations of AMP provoked by glucose deprivation, modifications of $P_{i}$ have little influence on the accumulation of adenosine (Fig. $5 e$ ), but at the lower AMP concentrations recorded under physiologic conditions and during alkalinization, a change in $\mathrm{P}_{\mathrm{i}}$, particularly its suppression, has a noticeable effect (Fig. 5 $d$ and $f$ ). A precise evaluation of the respective contributions of the changes in AMP and $P_{i}$ on the activity of the cytoplasmic 5 -nucleotidase will require studies on the purified enzyme. In addition, in intact erythrocytes incubated with glucose, the concentrations of AMP and $P_{i}$ cannot be varied totally independently from each other, because modifications of extracellular $P_{i}$ are accompanied by parallel changes in the rate of glycolysis (37, 38) resulting in opposite changes in the concentration of AMP.

The activity of AMP deaminase is reflected by the residual formation of hypoxanthine in the presence of $\mathrm{dCF}$ and ITu together. As evidenced by the 10 -fold higher rates of production of hypoxanthine recorded at the three concentrations of $\mathrm{P}_{\mathrm{i}}$ at alkaline (Fig. $5 c$ ) as compared to physiologic pH (Fig. $5 a$ ), the enzymic activity is also dependent upon the concentration of AMP. However, the much lower residual rates of production of hypoxanthine under glucose deprivation (Fig. $5 b$ ), when AMP is sixfold higher than in alkalinization, indicate that the concentration of AMP is not the only determinant of the activity of AMP deaminase. Erythrocytic AMP deaminase is known to be inhibited by 2,3-DPG and $P_{i}(3-6)$. Variations in the concentration of 2,3-DPG do not seem to play a major role in the catabolic conditions that were investigated. Indeed, 2,3-DPG was not modified at alkaline $\mathrm{pH}$ and although 2,3-DPG decreased by $25 \%$ in glucose deprivation, the residual production of hypoxanthine was severalfold lower under the latter condition than at alkaline $\mathrm{pH}$. Because a major difference between glucose deprivation and alkalinization is a threefold increase in intracellular $P_{i}$ in the first condition and a 50\% decrease in the second one, $P_{i}$ appears the most important effector of AMP deaminase. Because the production of hypoxanthine in the presence of $\mathrm{dCF}$ and ITu together in the absence of glucose was more dependent on the concentration of $\mathrm{P}_{\mathrm{i}}$ than that of adenosine, we may deduce that AMP deaminase is more sensitive to $P_{i}$ than the 5 -nucleotidase. Our results also indicate that the depletion of erythrocytic ATP reported in hypophosphatemic patients (14) and dogs (16) and the increase in red cell ATP prevailing in hyperphosphatemic uremic subjects (15) are, at least in part, due to modifications of the physiologic inhibition of AMP deaminase.

A substrate cycle between AMP and adenosine is known to operate in isolated rat hepatocytes under basal conditions, with a velocity that reaches about twice the rate of production of allantoin, the terminal purine catabolite in these cells (1). The two constitutive enzymes of the cycle, cytoplasmic 5'-nucleotidase and adenosine kinase, are also present in human erythrocytes. However, we have found no evidence that the cycle operates in these cells under normal conditions. Nevertheless, we have shown that some adenosine is formed and rephosphorylated when the concentration of AMP is increased, i.e., during glucose deprivation and alkalinization. During glucose deprivation, a condition characterized by a 30-fold elevation of AMP, a marked depletion of ATP, and a fast rate of production of adenosine ( $70-80 \mathrm{nmol} / \mathrm{h}$ per $\mathrm{ml}$ of packed cells), only $\sim 25 \%$ of this production is recycled to AMP by adenosine kinase in the absence of dCF. This is indicated by the effect of ITu alone to increase the production of hypoxanthine by $20 \mathrm{nmol} / \mathrm{h}$ per ml of packed cells. During alkalinization, a situation characterized by a less pronounced elevation of AMP and depletion of ATP, adenosine is produced at the rate of only $20-25 \mathrm{nmol} / \mathrm{h}$ per $\mathrm{ml}$ of packed cells and can be completely rephosphorylated. Recycling of adenosine can thus be considered a mechanism to protect the erythrocytic adenine nucleotide pool against degradation. Its efficiency is, however, limited since it can only keep pace with $10-15 \%$ of the rate of catabolism provoked by glucose deprivation, and with $\sim 30 \%$ of that induced by alkalinization. As evidenced by the small accumulation of adenosine in the presence of dCF and ITu (Fig. $5 d$ ), some recycling of the nucleotide also occurs in phosphate-free medium at physiologic $\mathrm{pH}$ in the presence of glucose.

Taken together our results demonstrate the existence of two alternative reactions for the initial degradation of AMP in human erythrocytes, i.e., deamination and dephosphorylation. Deamination appears more sensitive to $\mathrm{P}_{\mathrm{i}}$, whereas dephosphorylation is mainly responsive to variations of AMP. Recycling of adenosine does not occur under physiologic conditions, but can be evidenced when AMP catabolism is induced by glucose deprivation or by alkalinization.

\section{Acknowledgments}

The authors wish to express their appreciation to Mrs. Anne Delacauw for expert technical assistance. 
This work was supported by grant 3.4563 .82 of the Fonds National de la Recherche Médicale. Dr. Van den Berghe is Maître de Recherches of the Belgian Fonds National de la Recherche Scientifique.

\section{References}

1. Bontemps, F., G. Van den Berghe, and H. G. Hers. 1983. Evidence for a substrate cycle between AMP and adenosine in isolated hepatocytes. Proc. Natl. Acad. Sci. USA. 80:2829-2833.

2. Watts, R. W. E., J. E. M. Watts, and J. E. Seegmiller. 1965. Xanthine oxidase activity in human tissues and its inhibition by allopurinol (4-hydroxypyrazolo[3,4-d]pyrimidine). J. Lab. Clin. Med. 66:688-697.

3. Askari, A. 1966. Modifying effects of anions on the alkali-cationactivated AMP deaminase of human erythrocyte. Mol. Pharmacol. 2: 518-525.

4. Askari, A., and S. N. Rao. 1968. Regulation of AMP deaminase by 2,3-diphosphoglyceric acid: a possible mechanism for the control of adenine nucleotide metabolism in human erythrocytes. Biochim. Biophys. Acta. 151:198-203.

5. Lian, H. Y., and D. R. Harkness. 1974. The kinetic properties of adenylate deaminase from human erythrocytes. Biochim. Biophys. Acta. 341:27-40.

6. Yun, S. L., and C. H. Suelter. 1978. Human erythrocyte 5'-AMP aminohydrolase. Purification and characterization. J. Biol. Chem. 253: 404-408.

7. Valentine, W. N., K. Fink, D. E. Paglia, S. R. Harris, and W. S. Adams. 1974. Hereditary hemolytic anemia with human erythrocyte pyrimidine 5'-nucleotidase deficiency. J. Clin. Invest. 54:866-879.

8. Paglia, D. E., and W. N. Valentine. 1975. Characteristics of a pyrimidine-specific 5'-nucleotidase in human erythrocytes. J. Biol. Chem. 250:7973-7979.

9. Henderson, J. F., G. Zombor, P. W. Burridge, G. Barankiewicz, and C. M. Smith. 1979. Relationships among purine nucleoside metabolism adenosine triphosphate catabolism, and glycolysis in human erythrocytes. Can. J. Biochem. 57:873-878.

10. Mills, G. C., R. M. Goldblum, and F. C. Schmalstieg. 1981. Catabolism of adenine nucleotides in adenosine deaminase deficient erythrocytes. Life Sci. 29:1811-1820.

11. Mills, G. C., and L. B. Summers. 1959. The metabolism of nucleotides and other phosphate esters in erythrocytes during in vitro incubation at $37^{\circ} \mathrm{C}$. Arch. Biochem. Biophys. 84:7-14.

12. Mills, G. C. 1966. Effects of pH on erythrocyte metabolism. Arch. Biochem. Biophys. 117:487-493.

13. Schraufstatter, I., and G. V. R. Born. 1981. Dependence of purine loss from human erythrocytes on external pH. Br. J. Haematol. 49:349354.

14. Lichtman, M. A., D. R. Miller, and R. B. Freeman. 1969. Erythrocyte adenosine triphosphate depletion during hypophosphatemia in a uremic subject. N. Engl. J. Med. 280:240-244.

15. Lichtman, M. A., and D. R. Miller. 1970. Erythrocyte glycolysis, 2,3-diphosphoglycerate and adenosine triphosphate concentration in uremic subjects: relationship to extracellular phosphate concentration. J. Lab. Clin. Med. 76:267-279.

16. Yawata, Y., R. P. Hebbel, S. Silvis, R. Howe, and H. Jacobs. 1974. Blood cell abnormalities complicating the hypophosphatemia of hyperalimentation: erythrocyte and platelet ATP deficiency associated with hemolytic anemia and bleeding in hyperalimented dogs. J. Lab. Clin. Med. 84:643-653.

17. Van den Berghe, G., F. Bontemps, and H. G. Hers. 1980. Purine catabolism in isolated rat hepatocytes. Influence of coformycin. Biochem. J. 188:913-920.

18. Vincent, M. F., G. Van den Berghe, and H. G. Hers. 1982. The pathway of adenine nucleotide catabolism and its control in isolated hepatocytes subjected to anoxia. Biochem. J. 202:117-123.

19. Henderson, J. F., L. Brox, G. Zombor, D. Hunting, and C. A. Lomax. 1977. Specificity of adenosine deaminase inhibitors. Biochem. Pharmacol. 26:1967-1972.

20. Henderson, J. F., A. R. P. Paterson, I. C. Caldwell, B. Paul, M. C. Chan, and K. F. Lau. 1972. Inhibitors of nucleoside and nucleotide metabolism. Cancer Chemother. Rep. Part II. 3:71-85.

21. Krebs, H. A., and K. Henseleit. 1932. Untersuchungen über die Harnstoffbildung im Tierkörper. Hoppe-Seyler's Z. Physiol. Chem. 210: 33-66.

22. Klingenberg, M., and E. Pfaff. 1967. Means of terminating reactions. Methods Enzymol. 10:680-684.

23. Whelan, J. M., and A. S. Bagnara. 1979. Factors affecting the rate of purine ribonucleotide dephosphorylation in human erythrocytes. Biochim. Biophys. Acta. 563:466-478.

24. Hartwick, R. A., and P. R. Brown. 1975. The performance of microparticle chemically-bonded anion-exchange resins in the analysis of nucleotides. J. Chromatogr. 112:651-662.

25. Crabtree, G. W., and J. F. Henderson. 1971. Rate-limiting steps in the interconversion of purine ribonucleotides in Ehrlich ascites tumor cells in vitro. Cancer Res. 31:985-991.

26. Itaya, K., and M. Ui. 1966. A new micromethod for the colorimetric determination of inorganic phosphate. Clin. Chim. Acta. 14:361366.

27. Gawehn, K., and H. U. Bergmeyer. 1974. D-(-)Lactate. In Methods of Enzymatic Analysis. 2nd ed. H. U. Bergmeyer, editor. Academic Press, Ltd., London. 1492-1495.

28. Michal, G. 1974. D-glycerate-2,3-diphosphate. In Methods of Enzymatic Analysis. 2nd ed. H. U. Bergmeyer, editor, Academic Press, Ltd., London. 1433-1438.

29. Michal, G., and H. O. Beutler. 1974. D-fructose-1,6-diphosphate, dihydroxyacetone phosphate and D-glyceraldehyde-3-phosphate. In Methods of Enzymatic Analysis. 2nd ed. H. U. Bergmeyer, editor, Academic Press, Ltd., London. 1314-1319.

30. Rapoport, I., S. Rapoport, and R. Elsner. 1981. Accumulation of phosphate esters and decline of ATP in red cells incubated in vitro is caused by lack of pyruvate. Acta Biol. Med. Germ. 40:115-121.

31. Minakami, S., S. Takayasu, C. Suzuki, and H. Yoshikawa. 1964. The hydrogen ion concentrations and erythrocyte glycolysis. Biochem. Biophys. Res. Commun. 17:748-751.

32. Albrecht, V., H. Roigas, M. Schultze, G. Jacobasch, and S. Rapoport. 1971. The influence of $\mathrm{pH}$ and methylene blue on the pathways of glucose utilisation and lactate formation in erythrocytes of man. Eur. J. Biochem. 20:44-50.

33. Van den Berghe, G., M. Bronfman, R. Vanneste, and H. G. Hers. 1977. The mechanism of adenosine triphosphate depletion in the liver after a load of fructose. A kinetic study of liver adenylate deaminase. Biochem. J. 162:601-609.

34. Van den Berghe, G., C. van Pottelsberghe, and H. G. Hers. 1977. A kinetic study of the soluble 5 '-nucleotidase of rat liver. Biochem. $J$. 162:611-616.

35. Carson, D. A., and D. B. Wasson. 1982. Characterization of an adenosine 5'-triphosphate- and deoxyadenosine 5'-triphosphate-activated nucleotidase from human malignant lymphocytes. Cancer Res. 42:43214324.

36. Bontemps, F., G. Van den Berghe, and H. G. Hers. 1985. Identification of a purine-specific 5'-nucleotidase in human erythrocytes. $\mathrm{Pe}$ diatr. Res. 19:747. (Abstr.)

37. Rose, I. A., J. V. B. Warms, and E. L. O'Connell. 1964. Role of inorganic phosphate in stimulating glucose utilization of human red blood cells. Biochem. Biophys. Res. Commun. 15:33-37.

38. Tsuboi, K. K., and K. Fukunaga. 1965. Inorganic phosphate and enhanced glucose degradation by intact erythrocytes. J. Biol. Chem. 240: 2806-2810. 